\title{
Artificial Intelligence in Healthcare
}

\author{
Saiful Islam

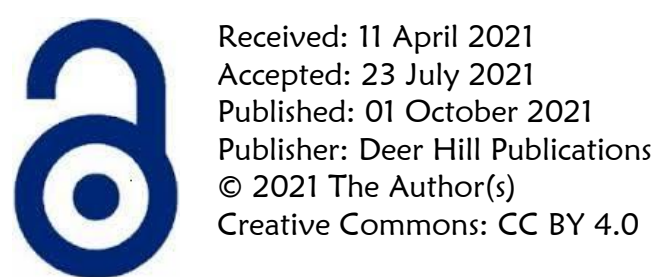

\begin{abstract}
Artificial intelligence $(\mathrm{Al})$ is the ability of a computer program or machine to think or learn that possess human-like intelligence. These computing devices use this intelligence to provide services such as speech recognition, natural language processing and identifying disease in healthcare. To work efficiently, Al requires adequate data that is used to train systems. The efficiency of any Al system depends on the availability of this data. This article is mainly focused on recent advents in the technology of Artificial Intelligence. The importance of Al in healthcare is identified and described in this report. The applications of Artificial Intelligence in healthcare such as clinical care, medical research, drug research and public healthcare are briefly discussed here. The purpose of this article is to demonstrate that artificial intelligence is being used in all domains of life and particularly in the field of healthcare. This report presents the role of Artificial Intelligence in healthcare.
\end{abstract}

Keywords: Artificial Intelligence, Neural Network, Machine Learning, Healthcare, Medicine, Diagnosis.

\section{INTRODUCTION}

Artificial intelligence deals with the computer or any computing resources that perform some tasks which usually requires human-like intelligence. Al has enabled significant progress in natural language processing, speech recognition, image processing, computational intelligence, and many other areas. All these advances in computer science open many opportunities in artificial Intelligence by enhancing human decision making in healthcare. Recent research and studies show that $\mathrm{Al}$ can perform medical and clinical diagnostics at a level equal to experienced clinicians. Al can be integrated and applied to many healthcare applications including detection of disease, delivery of health services and drug discovery.

Al has the potential to help address important healthcare challenges, but the efficiency of Al system depends on the availability of quality health data which is used to train system. At the same time, health data has many privacy issues associated with it, collecting health data of the patient, and sharing those data to user is practically difficult task and quite expensive. There are many private sector companies which are collecting and selling those data.

Some major applications of $\mathrm{Al}$ in healthcare organizations are described below:

\section{A. Medical Research}

Al can be used to analyse and identify patterns from large complex datasets faster than compared to any other previously developed systems. Al can also be applied for searching scientific articles for research and studies. Researchers have also developed an artificial intelligence-based robo-scientist called EVE which is designed to make drug discovery faster than ever.

\section{B. Healthcare Organization}

Al can be used to improve the patient experience which can be in the form of an app which facilitates interaction with patients. This app collects appropriate information from patients and delivers to clinicians that help them to deliver appropriate treatment.

\section{Clinical Care}

Al systems help in identifying diseases by analysing clinical data and research publications. Possible uses of $\mathrm{Al}$ in clinical care includes:

\section{S. Islam}

MASTEQ Software Pty Ltd

Collins Street Tower, Level 3, 480 Collins Street

Melbourne, VIC 3000, Australia

E-mail: saiful.islam@masteq.com.au

Reference: Islam, S. (2021). Artificial Intelligence in Healthcare. International Journal of Engineering Materials and Manufacture, 6(4), 319-323. 
I. Medical imaging: Al shows promising results in detecting diseases such as breast and skin cancers, eye diseases and other medical implications by systematically collecting medical scans and other health records.

II. Echocardiography: Using ultrasonic systems integrated with Al helps in detecting patterns of heartbeats and diagnose coronary heart disease. Such a system is implemented at John Radcliffe Hospital in Oxford.

III. Surgery: Robotic arms controlled by Al are used in clinical surgery.

\section{Public Health}

Al has the potential to identify infectious diseases with their cause sources like water contamination, air pollutants, and others. Al also helps in identifying adverse drug effects by scanning drug content.

\section{E. Patient and Consumer-facing Applications}

Several apps are available in markets that offer healthcare advice and personalized health assessments. These apps identify medical implications by communicating with the patient in the form of a chatbot.

Artificial intelligence-based systems have some limitations based on where it is applied. Al in healthcare also has some limitations which are listed below.

i. Digital health data is required to train ai based systems and apps to maintain the consistency of this digital data.

ii. Humans have some attributes that are difficult for Al to process such as compassion.

\section{RESEARCH METHODOLOGY}

The research article is secondary in nature. The author has analysed empirical works of celebrated professionals and respected organizations to find the outcome of this research paper.

\section{AI TECHNOLOGY IN HEALTHCARE}

Following are the major fields in which Artificial Intelligence can aid modern Health Care \& recent Projects which are being implemented.

\subsection{Using Artificial Intelligence to Improve Hospital Inpatient Care}

According to Daniel B. Neill (2005), Al has the potential to improve many patients' care processes. Integrating Al in healthcare will maximize the efficiency of the healthcare system and minimize the risk of side effects by recommending appropriate diagnostic tests. Al uses previous health data to discover new medical knowledge, it also monitors the patient's health to collect train dataset which ultimately increases system performance. In this paper, the author suggested one way to collect data of health records using Electronics Health Records (EHR) as per the Health Information Technology for Economic and Clinical Health (HITECH) Act. The author shows recent advances in Al system by showing case studies of various healthcare systems. Clinical decision support systems (CDSS) are one of the most successful healthcare applications which primarily focus on the diagnosis of a patient's condition by giving symptoms and other demographic information as input to system. Artificial Intelligence is combined with machine learning to detect and identify diseases based on large pool of healthcare data. Artificial Intelligence analyses past data and learns the pattern. It analyses all the cases of disease and learns about the symptoms of the disease. It then creates filters which can alert prior to shock \& organ dysfunction. Artificial Intelligence helps doctors by providing pre-diagnosis and assist them to move forward to the right direction of treatment when a patient visits the hospital. Several inhibitors of beta-Secretase are used for the treatment of Alzheimer's. A machine learning (ML) algorithm is developed considering structural, physical and chemical characteristics of these inhibitors. ML, Multilayer Perceptron (MLP), Logitboost (LB), \& Decision Table (DT) functions were applied to set of Molecular Descriptors for an active and inactive compound in 5 -fold cross validation. The highest accuracy achieved by this data set was $88.86 \%$ by MLP. The developed model is currently being used for selection of possible drug candidate from the database of these compounds.

\subsection{Primary Healthcare Using Artificial Intelligence}

Khairnar, V. D., Saroj, A., Yadav, P., Shete, S. \& Bhatt, N. (2019) has proposed the new technology which emphasizes in providing proper health care to the remote patients by keeping in mind their poor financial conditions, lack of facilities and lack of means of transportation by prescribing them the precise medicine within a millisecond excluding the communication delay with the help of artificial neural network (ANN) by converting the received voice messages from the patient to text message, and in replay to their solution the medicines are also sent by text messages. The technology includes Neural Network, Natural Language Toolkit, Google API, Natural Language Processing, Programming Language-Python, Interactive Voice Response, PHP, HTML, R Studio and Artificial Neural Network. The main objective of this technology is to collect the data related to various diseases and medicines to cure the disease to create an environment that focuses on the high quality of patient health care. Using this technology, they enhance the quality of health care and bridge the gaps in healthcare services, which significantly saves the life of the person in the absence of the doctor. Recently doctors developed an Algorithm with the help of Google to detect the cardiovascular risks to a person by recognizing hidden patterns in Retinal images. More than 280,000 patients' Retinal images were utilised to train and understand the hidden pattern [3]. It was verified on two distinct datasets of 12,026 \& 999 patients, respectively. Another machine makes out the peculiarities of a blood vessel and inform different 
variables such as age (+/- 3.3 yrs.), smoking status, systolic blood pressure, and HbA1c by analysing images. Detection of Diabetic Retinopathy \& Macular edema was automated just by Analysing Retinal fundus photographs at the same way.

\subsection{Mobile Based Healthcare Management using Artificial Intelligence}

To propose the proper health care management system, Amiya, K., Tripathy, A.K., Carvalho, R., Pawaskar, K., Suraj Y. and Vijay, Y. (2015, pp 1-6) proposed the system which consists of mobile-based heart rate management so that the data can be transferred and diagnosed based on the heart rate and communicated directly to the doctor. It also includes the video conferencing facility which helps to communicate face-to-face with the doctor and get out of the problem, the system consists of Doc-Bot module which gives solution to the disease of the patient and the module is implemented in Artificial Intelligence Markup Language (AIML), the Doc-Bot uses feed-forward neural network for classification of the disease and solving them. The proposed system also consists of the Online Blood Bank which provides the latest details related to the type and quantity of the availability of the blood in different hospitals.

\section{3,4 Mapping the Challenges of Artificial Intelligence in the Public Sector}

Sun, T. Q. \& Medaglia, R. (2019) focused on adaption of the Artificial Intelligence (AI) System in IBM Watson in public healthcare to map to analyse how the three groups of stakeholders (Government policymakers, hospital managers/doctors and Information Technology (IT) firm managers) receive the challenges of Artificial Intelligence (Al) in public sector. Artificial Intelligence is the most potential in doing transformative work - mining medical records, assisting repetitive jobs and designing treatment plans. The system focuses on huge data collection related to the various diseases and their treatment, huge collection of books, articles, and notes related to various medicines so that no disease should be left out which does not have its treatment. Machine Learning governs and trains complicated Neural Networks such as IBM Watson Cloud \& Targeted Realtime Early Warning System (T.R.E.W.S.) [17]. These sophisticated systems analyse age, BMI, heart rate, exercise habits, eating habits, sleep pattern and human's psychological behaviour through various sensors such as smartwatch, health band, browsing history, mobile Phone activity etc. Once the data is uploaded on the cloud, this data is categorized and analysed with the help of Cognitive Networks.

\subsection{Artificial Intelligence Image Recognition in Healthcare.}

Gavrilov, D., Melerzanov, A., Schelkunov, N. \& Gorodilov, A. (2018) focused on one of the most dangerous cancer tumors - melanoma. As melanoma does not have the painful symptoms in the early stage so the cancer is diagnosed late. The proposed system states the using of the computer vision to solve the problem of detecting melanoma at an early stage. After the introduction of such a diagnosis, it becomes possible for the expert to take a proper decision and based on that decision various tools are used such as telemedicine. The system is based on the deep convolutional neural network, it uses ImageNet Large Scale Visual Recognition Challenge (ILSVRC) which provides proper image of the disease. They used the ImageNet Challenge to prepare inception of the disease. The system also proposed the online skin check-up so that every user can check his/her moles for melanoma risk online without any cost. The system success rate is $91 \%$ of recognition of skin disease.

\subsection{Artificial Intelligence Contribution to eHealth Application.}

Cabestany, J., Rodriguez-Martín, D., Pérez, C., \& Sama, A. (2018) proposed the system to analyse how Artificial Intelligence ( $\mathrm{Al}$ ) technique can improve the management of data generated by the eHealth activity. In this system, they have developed a wearable device that allows continuous monitoring of the patient's disease. This new invented device "Holter" will help neurologists to diagnose more accurately the disease of the patient and let them to take critical decisions about the medicine and the treatment related to the disease. This new wearable device consists of microcontroller to manage all peripherals, a set of sensing components, communication module-based Bluetooth technology and a power management system that can minimize the internal consumption during its execution period. The result detected by the wearable device is then analysed through a set of various algorithms to diagnose the disease and help to reach through its treatment. To understand how data is collected by this new computing device, they have proposed the four different levels of multi-layered architecture, which include - sensing layer, networking layer, service layer and interface layer which provides an interface to the user and other applications.

\subsection{Application of Artificial Intelligence to Cure Disease}

Chanchaichujit, J. (2015) proposed a system that is based on the control and management of Tuberculosis (TB) using Artificial Intelligence (AI). The author suggested that the TB disease is caused by M. Tuberculosis bacteria (MTB), this bacteria mainly affects the lungs and that TB is an airborne disease which spread through the air with the contact of MTB caused person. The author has given three stages to get controlled form TB - Diagnostic connectivity, Data repository and gateway, and Data representation and applications. The author conveys that one of the key success factors for developing the Artificial Intelligence ( $\mathrm{Al}$ ) for Tb detection is the training of the algorithm with a huge amount of the data, which will later help them to differentiate the infection from the normal cases. The author uses the DAC4TB, which consists of a huge amount of data to cure TB, which includes data of multiple TB tests, CXR, $A F B$, GeneXpert and TB cultures of many patients and various design algorithms to cure TB disease. With the help of 
such more comprehensive results, physicians and healthcare workers can make better recommendations. Artificial intelligence creates a valid clinical pathway to reduce the cost and time of traditional methods. Piyush Mathur \& Kamal Maheshwari conducted a study under the aegis of Ayasdi Clinical Variation Management (CVM) application where 1,786 patients were analysed [7]. All the patients had Colorectal Surgery in 2015-2016. The program analysed multidimensional data sets under supervision. Results segregated their data set into 9 different groups with Similarity Analysis based on Ketorolac dosage. This dosage is a Non-Steroidal Anti-inflammatory drug (NSAID) which is usually used in Surgery to treat postoperative pain. In addition, they identified a link to low intraoperative fluids and relatively lower length of stay along with lower cost group. This eventually broaden their clinical pathway for treatment. Artificial Intelligence aids as a Virtual Assistant in Robotic Technology. Fluoroscopic imaging guides to do Heart Catheterization that exposes operator, staff \& patient to harmful radiation. To avoid this radiation, a selfdirected robot (CATH-BOT) is used to remotely supervise cardiac catheterization [7]. This robot develops a patient's specific 3D anatomical model by hospital electronic record system that includes MRI, CT, and Ultrasound etc. Temporal syncing is implemented through Respiratory/ECG gating. Catheter's location is tracked via an electromagnetic tracking system with patient anatomy. All trial catheterizations were successfully completed and no difference in Fluoroscopy EMTs was found in initial result. Total procedural time was 3.9 secs lower than Fluoroscopy. This project is still under improvisation which will also add catheter feedback positioning.

\section{CONCLUSIONS}

In rapid healthcare advancement, it is necessary to adopt new changes in the system and deliver better services to patients. Al has incredible potential to learn from past data or experience and make better decisions in the future. Artificial intelligence is very useful in healthcare. Al, ML, and DL combining help in identifying diseases, allotting proper drugs to patients and reduces the risk of side effects of drugs. Al also helps in identifying real-time health information by continuously monitoring users' behaviours and emotions. This paper revealed different applications of $\mathrm{Al}$ in healthcare, which are the attributes that $\mathrm{Al}$ uses to detect medical implications, factors affecting the efficiency of the Al system. Al with machine learning algorithms helpful in tuberculosis detection, brain tumour detection and not only for detection we can also apply $\mathrm{Al}$ in medical diagnostic and clinical care. Many medical practitioners believe that technology has several advantages over traditional practices as it can analyse large datasets simultaneously, gives us an unsupervised discovery which discloses hidden patterns and enhances the speed by suggesting auto-generated clinical pathways. Artificial Intelligence is a tool which provides aid to medical practitioners in early diagnosis and help lower the mortality rate \& medical inflation. The most challenging issue in artificial intelligence is to collect health data and to identify medical implications from available data due to less amount and complexity of data. Al community is on the way to develop such algorithms that are more adequate in pattern matching and identification and able to work on a massive quantity of unstructured health data.

\section{REFERENCES}

1. Adam. C. \& Uzialko (2019). Artificial Intelligence Will Change Healthcare as We Know It. Business News Daily. Retrieved from https://www.businessnewsdaily.com/15096-artificial-intelligence-in-healthcare.html

2. Cabestany, J., Rodriguez-Martín, D., Pérez, C., \& Sama, A. (2018, June). Artificial Intelligence Contribution to eHealth Application. In 2018 25th International Conference" Mixed Design of Integrated Circuits and System"(MIXDES) (pp. 15-21). IEEE.

3. Cath-bot: first step toward an independent heart catheterization robot - AlMed [Internet]. AIMed. 2018 [cited 2 November 2018]. Available from: http://ai-med.io/dt_team/cath-bot-first-step-towardan-independent-heartcatheterization-robot/

4. Gavrilov, D., Melerzanov, A., Schelkunov, N., \& Gorodilov, A. (2018). Artificial Intelligence Image Recognition In healthcare. In 2018 International Conference on Artificial Intelligence Applications and Innovations (IC-AIAI) (pp. 24-26). IEEE.

5. Gulshan, V., Peng, L., Coram, M., Stumpe, M., Wu, D. \& Narayanaswamy, A. Development and Validation of a Deep Learning Algorithm for Detection of Diabetic Retinopathy in Retinal Fundus Photographs. JAMA. 2016; 316 (22): 2402

6. Henry, K., Hager, D., Pronovost, P., Saria, S. A targeted real-time early warning score (TREWScore) for septic shock. Science Translational Medicine. 2015;7(299):299ra122- 299ra122.

7. Identifying clinical variation using machine intelligence: A pilot in colorectal SURGERY - AIMed [Internet]. AIMed. 2018 [cited 2 November 2018]. Available from: http://ai-med.io/dt_team/identifying-clinicalvariationusing-machine-intelligence-a-pilot-incolorectal-surgery/

8. Khairnar, V. D., Saroj, A., Yadav, P., Shete, S., \& Bhatt, N. (2019). Primary Healthcare Using Artificial Intelligence. In International Conference on Innovative Computing and Communications (243-251). Springer, Singapore.

9. Furmankiewicz, M., Sołtysik-Piorunkiewicz, A. \& Ziuziański, A. (2014). Artificial intelligence systems for knowledge management in e-health: the study of intelligent software agents. Research gate. Retrieved from https://www.researchgate.net/profile/Anna_Soltysik- 
Piorunkiewicz/publication/271014570_Artificial_Intelligence_Systems_for_Knowledge_Management_in_eHealth_The_Study_of_Intelligent_Software_Agents/links/54bd0ee30cf218da9390d759/Artificial-IntelligenceSystems-for-Knowledge-Management-in-e-Health-The-Study-of-Intelligent-Software-Agents.pdf

10. Machine-learning models for selection of drugcandidates for treatment of alzheimer's disease - AIMed. AIMed. 2018 [2 November 2018]. Retrieved from: http://ai-med.io/dt_team/machine-learning-modelsfor-selection-ofdrug-candidates-for-treatment-ofalzheimers-disease/

11. Neill, D. B. (2013). Using artificial intelligence to improve hospital inpatient care. IEEE Intelligent Systems, 28(2), 92-95.

12. Nivetha, M. \& Nivethika, S. (2018). Artificial Intelligence in Healthcare - A Review. Research gate. Retrieved from https://www.researchgate.net/publication/329163470_Artificial_Intelligence_in_Healtshcare-A_Review

13. Peter, J. \& Kent, N. (2012). Patient behaviour and the benefits of artificial intelligence: The perils of " $\overline{\text { dangerous" }}$ literacy and illusory patient empowerment. Elsevier. Retrieved from https://www.researchgate.net/profile/Peter_Schulz2/publication/221250696_Bad_Literacy_the_Internet_and_t he_Limits_of_Patient_Empowerment/links/57f5422108ae91deaa5c77e9/Bad-Literacy-the-Internet-and-theLimits-of-Patient-Empowerment.pdf

14. Sun, T. Q. \& Medaglia, R. (2019). Mapping the challenges of Artificial Intelligence in the public sector: Evidence from public healthcare. Government Information Quarterly, 36(2), 368-383.

15. Thomas, M. \& Maddox. (2019). Questions for Artificial Intelligence in Health Care. Viewpoint. Retrieved from https://jamanetwork.com/journals/jama/article-abstract/2718456

16. Tripathy, A. K., Carvalho, R., Pawaskar, K., Yadav, S. \& Yadav, V. (2015, February). Mobile based healthcare management using artificial intelligence. In 2015 International Conference on Technologies for Sustainable Development (ICTSD) (pp. 1-6). IEEE.

17. Vincent, J. The Clinical Challenge of Sepsis Identification and Monitoring. PLOS Medicine. 2016;13(5): e1002022.

18. Zillner, S. \& Neururer, S. Big Data in the Health Sector. New Horizons for a Data-Driven Economy. 2016:179194.

19. 2017 Global Medical Trends Survey Report [Internet]. Willis Towers Watson. 2018 [cited 2 November 2018]. Retrieved from: https://www.willistowerswatson.com/en/insights/2 017/05/2017-global-medical-trendssurvey-report 